\title{
Control of rheumatoid arthritis with conventional disease-modifying antirheumatic drugs in a tertiary hospital in central South Africa
}

\author{
R M N Carter, ${ }^{1}$ FCP (SA), MMed (Internal Medicine); B J Jansen van Rensburg, ${ }^{1}$ FCP (SA), MMed (Internal Medicine), \\ Cert Rheumatology (SA); G Joubert, ${ }^{2}$ BA, MSc \\ ${ }^{1}$ Department of Internal Medicine, Faculty of Health Sciences, University of the Free State, Bloemfontein, South Africa \\ ${ }^{2}$ Department of Biostatistics, Faculty of Health Sciences, University of the Free State, Bloemfontein, South Africa
}

Corresponding author: R M N Carter (carterrmn@ufs.ac.za)

\begin{abstract}
Background. Rheumatoid arthritis (RA) is a highly prevalent disease with a significant impact on morbidity and life expectancy. There is a paucity of literature relating to the current state of disease control in South Africa, and none on Free State Province.

Objectives. To evaluate control of RA with conventional disease-modifying antirheumatic drugs (DMARDs) in the rheumatology outpatient department of Universitas Academic Hospital, Bloemfontein, and to determine the relative impact of various factors contributing to the prevention of disease control.

Methods. A cross-sectional study was undertaken over a period of 8 months from December 2016 to August 2017 in the rheumatology outpatient department of Universitas Academic Hospital. Data were collected by means of information sheets and questionnaires completed by attending doctors. The information pertained to disease activity, possible factors contributing to poor control, and relevant demographic data. Results. Information was collected from 169 patients, and data analysis was performed on 161. The results revealed that RA was controlled in 34 patients (21.1\%). Of the 127 patients with uncontrolled disease, 61 (48.0\%) reported dispensing issues relating to poor drug availability. Seventy-two (56.7\%) of the patients with uncontrolled disease were deemed to be on insufficient treatment for their disease state, of whom $33(45.8 \%)$ also reported concomitant dispensing issues. Other factors such as transport/access problems, administrative issues, adverse events and poor compliance/insight contributed to the inadequate control of RA to a minor extent. In terms of monthly dispensing, 159 patients (98.8\%) reported receiving all their DMARDs during the first month. The vast majority of these drugs were dispensed from Universitas Hospital. This figure decreased to 119 patients (73.9\%) who received all their DMARDs during the second month, mostly from district units. Only 55.3\% $(n=89)$ of the patients reported receiving all of their DMARDs from their down-referral units.

Conclusions. Control of RA at this institution is suboptimal compared with national and international standards. The main contributors to poor control appear to be problems relating to dispensing of medication and inadequate escalation of therapy by doctors. Most of the concern with the dispensing of medication lies with the poor availability of DMARDs in peripheral unit pharmacies. These factors are remediable and should be attended to.
\end{abstract}

S Afr Med J 2020;110(1):27-31. https://doi.org/10.7196/SAMJ.2020.v110i1.14099

Rheumatoid arthritis (RA) is a common condition with a global prevalence of $\sim 1 \%{ }^{\left[{ }^{[1]}\right.}$ African-based prevalence studies are sparse with heterogeneous findings, currently suggesting a slightly lower frequency. However, further research is required to ascertain the prevalence of RA in Africa. ${ }^{[2,3]}$ The disease is associated with decreased life expectancy and impaired quality of life, and places a significant financial burden on patients and society at large. ${ }^{[4]}$ Numerous developments in the management of RA have recently emerged. Most notable is the importance of early aggressive management according to a 'treat-to-target' approach, and the introduction of biological disease-modifying antirheumatic drugs (bDMARDs). ${ }^{[5,6]}$ These drugs are highly effective with regard to disease control, delaying clinical and radiological progression and joint destruction, and improving quality of life. ${ }^{[7]}$

South African (SA) guidelines for the management of RA were published in 2013. ${ }^{[6]}$ Drafted by the South African Rheumatism and Arthritis Association, these guidelines were compiled in accordance with international standards of treatment. The guidelines advocate early use of conventional synthetic disease-modifying antirheumatic drugs (csDMARDs) and rapid escalation of therapy, with the goal of reaching a state of low disease activity (LDA) or preferably remission. The csDMARDs typically used include methotrexate, sulphasalazine, chloroquine (or hydroxychloroquine) and leflunomide. ${ }^{[6]}$ Methotrexate remains the anchor of treatment with these agents, with other drugs being included by means of an add-on approach or sequential monotherapy. ${ }^{[8]}$ According to these guidelines, patients should be considered for initiation on a biological agent if the disease is not controlled to target (LDA or remission) by 6 months despite adequate escalation in treatment. ${ }^{[6]}$ Significant cost is involved in the procurement of biological agents, and the use of these drugs is therefore very limited in the public sector. Consequently, the vast majority of patients remain dependent on conventional agents.

Research has shown that the degree of disease control on traditional DMARDs varies significantly and depends on various factors, such as duration of disease and the scoring system used to determine disease activity. A study in Johannesburg comparing disease activity scores revealed that $>60 \%$ of patients were in a state of either LDA or remission, with minimal variation between the scoring tools. ${ }^{\left[{ }^{[9}\right.}$ This finding was comparable to Turkish data. ${ }^{[10]}$ In 2012, however, Hodkinson et al. ${ }^{[11]}$ found that only $28.4 \%$ of patients with early RA 
achieved LDA/remission after 12 months of traditional DMARDs, which was again comparable to other international data. Despite the impression of generally poor control of RA, data relating to the degree of disease control in SA are limited, and there are none for Free State Province. Numerous reports from patients and healthcare providers have suggested a lack of drug availability at peripheral pharmacies. As poor disease control has a profoundly negative impact, it is important to determine to what degree patients' RA is controlled, and to elucidate any factors preventing control. Addressing these issues will assist with improvement in patient management, especially in this resource-limited setting where more advanced treatments are not readily available.

\section{Objectives}

To evaluate the degree of disease control of RA with conventional DMARDs in the rheumatology outpatient department of Universitas Academic Hospital in Bloemfontein, SA, and to determine the relative impact of various factors contributing to the prevention of disease control.

\section{Methods \\ Study design and setting}

This cross-sectional study was conducted in the rheumatology outpatient department of Universitas Academic Hospital during the arthritis clinics on Mondays and Thursdays where all patients with RA are seen. The department manages the majority of patients with RA in Bloemfontein's public healthcare sector, as well as a large portion from the rest of the Free State and a number from Northern Cape Province. The department serves as the only rheumatology department in the Free State and Northern Cape provinces.

\section{Participants}

Participants deemed eligible for inclusion were patients with RA fulfilling the American College of Rheumatology/European League Against Rheumatism criteria of 2010 (as per the SA RA guidelines) who had been treated for at least 6 months, thus ensuring sufficient time for adequate escalation of therapy by the time of enrolment. ${ }^{[6]}$ Patients were selected consecutively until a predetermined minimum of 150 participants were included. Patients on biological agents and those with inadequate or unclear information crucial to the primary or secondary objectives of the study were excluded from the study.

\section{Data collection}

Before each clinic, patient files were screened for participants eligible for enrolment. These patients were then handed information documents with a detailed description of the intended study and proceedings to follow. After discussing all aspects of the study with each patient, written informed consent was obtained. An information sheet and questionnaire were completed by the attending doctor or the researchers during consultations. A copy was kept in each source file to prevent rescreening of the same patient.

The following data were collected on the information sheets: (i) from the source file: basic demographic data, the Clinical Disease Activity Index (CDAI) score, what DMARDs the patient was taking at the time of the study, and the dosage; and (ii) what pharmacies the patient had attended each month (viz. 1st, 2nd and 3rd - 6th months), whether they were receiving all their DMARDs every month (and if not, which agents they had not received, and for what reason), and whether the patient was taking all the DMARDs received (and if not, whether this was due to compliance/insight problems, adverse drug reactions, or any other reason). Patients' reasons for not taking all their medications were completed by ticking the relevant boxes. Additional space was provided for elaboration on unforeseen factors. Patients were requested pertinently to provide information with regard to taking of medication and reasons for not taking their medication as prescribed.

Disease control was assessed by means of the CDAI score, an internationally accepted composite scoring system comprising four parameters to determine overall disease activity, namely: (i) a swollen joint count; (ii) a tender joint count; (iii) the patient's global assessment; and (iv) the doctor's global assessment of the disease state $^{[7]}$ As opposed to other validated scoring systems, the CDAI score was used in this study because it is the current standard of assessment used in this institution, and the accuracy is comparable to that of other validated disease activity scores. ${ }^{[9]}$ The degree of disease control was grouped into four categories based on the patient's CDAI score, namely: (i) $\leq 2.8=$ remission; (ii) 2.9 - 10 = LDA; (iii) $11-22=$ moderate disease activity; and (iv) $>22=$ high disease activity. A score falling within the categories of LDA or remission was considered to indicate disease control.

The minimal acceptable treatment for a patient with uncontrolled disease was considered to be at least three csDMARDs at optimally high doses, defined in this study as follows: methotrexate at least $20 \mathrm{mg}$ weekly, sulphasalazine at least $1 \mathrm{~g}$ twice daily, chloroquine at least $200 \mathrm{mg}$ daily, and leflunomide at least $20 \mathrm{mg}$ daily. In a situation where an adverse event caused by a DMARD was the only factor preventing further escalation of therapy, the treatment would still be deemed sufficient. In cases where the disease was not controlled despite the absence of any possible explanation for poor control, it was considered to be refractory to csDMARDs.

The data collected were transferred to a Microsoft Excel spreadsheet for analysis. The various factors identified as possible contributors to poor control were grouped as follows: (i) insufficient treatment for disease state; (ii) dispensing problems; (iii) adverse effects of medication; (iv) poor compliance/insight; ( $v$ ) administrative problems; ( $v i)$ problems involving access/transport; and (vii) other unforeseen issues.

\section{Statistical analysis}

Data were analysed by the Department of Biostatistics of the University of the Free State using the Statistical Analysis System version 9.3 (SAS Institute, USA). Results were summarised using frequencies and percentages for categorical variables, and percentiles for numerical variables due to skew distributions.

\section{Ethical considerations}

Ethical approval was obtained from the Health Sciences Research Ethics Committee of the University of the Free State (ref. no. HSREC 108/2016) prior to data collection. Provincial permission was then granted by the Free State Department of Health (ref. no. FS_2016RP3_140). Written informed consent was obtained from all participants enrolled in the study.

\section{Results}

Data were collected from 169 participants over a period of 8 months. Of the 169 participants, 8 were excluded from data analysis, 6 on the basis of insufficiently completed information sheets and 2 due to current or planned pregnancy. The authors considered the latter to be a confounding factor in that pregnancy has an effect on disease control and pregnant patients cannot use certain DMARDs owing to teratogenic effects. Data analysis was performed on the remaining 161 participants (Fig. 1). 


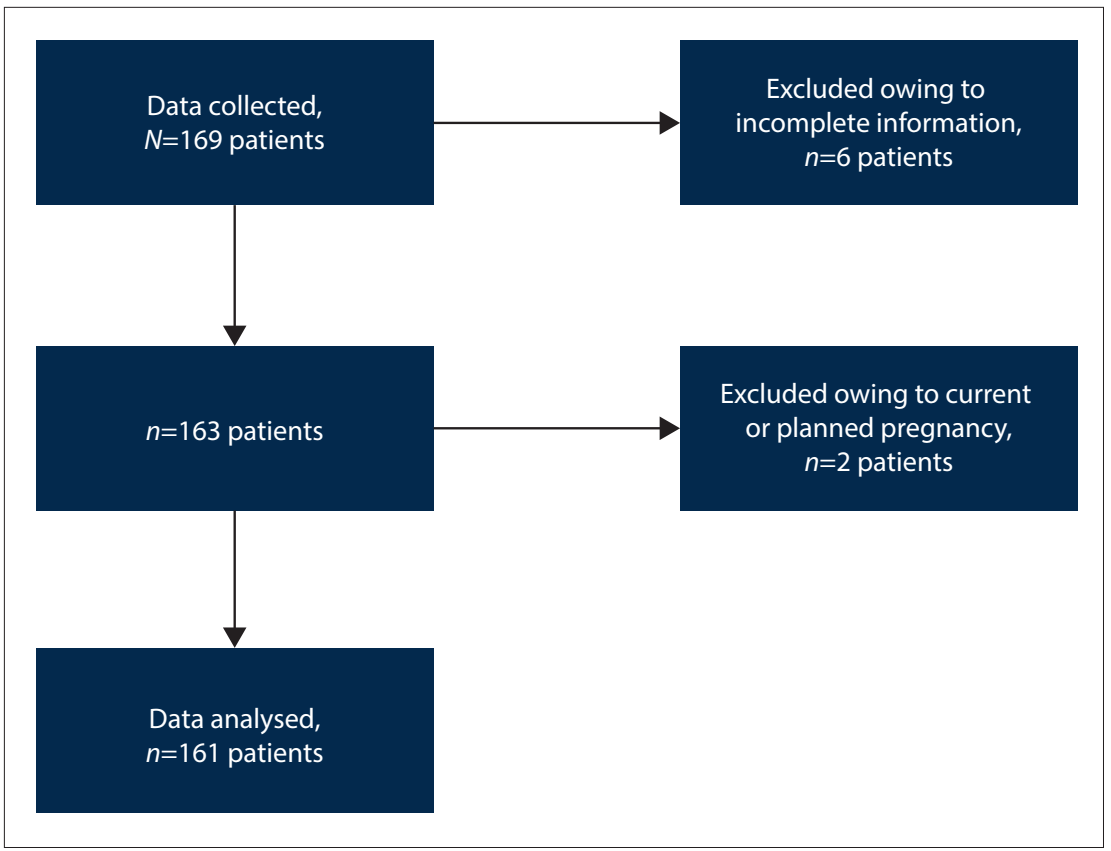

Fig. 1. Enrolment of participants.

The study had a well-represented sample population, as shown by the demographic information set out in Table 1. The patients' median age was 58 years (range $19-83$ ). The majority of patients were female $(n=138,85.7 \%)$ and black African $(n=131$, $81.4 \%)$. With regard to degree of disease control (Table 1), 34 (21.1\%) were optimally controlled and only $5(3.1 \%)$ were in remission. In contrast, 71 patients $(44.1 \%)$ with high disease activity were identified.

Table 2 summarises the various factors reported by patients that may have contributed to poor disease control in the total study population, patients with an uncontrolled disease state, and patients in whom the disease was deemed to be controlled. In $21(13.0 \%)$ of the total group of patients, no discernible cause for poor control was found, and these patients were considered to have disease that was refractory to csDMARDs.

Of the 34 patients with controlled RA, $24(70.6 \%)$ had no documented factors that might have impacted on their disease control, and $10(29.4 \%)$ were controlled despite possible factors that might have resulted in poor control.

With regard to the dispensing of DMARDs, 159 of the participants (98.8\%) reported receiving all of their medication for their first month of treatment, and 154 (95.7\%) received their medication from the Universitas Hospital pharmacy. As shown in Fig. 2, the number of patients receiving all of their DMARDs decreased over time. From the second month onwards, patients mainly received their medication from different healthcare facilities in the five districts of the Free State.

\section{Discussion}

The study revealed that disease control of RA at Universitas Academic Hospital is suboptimal compared with available national and international data, with other studies showing a degree of control ranging between $28 \%$ and $60 \% \cdot{ }^{[9-13]}$ However, studies reporting control are limited and further research is required on provincial and national levels.

Poor control could be attributed to contributing factors such as inadequate escalation of treatment and problems with the dispensing system, resulting in lack of availability of medication, mainly at peripheral unit pharmacies.

A large proportion of patients seemed to be on inadequate treatment for their disease state. It should be noted, however, that common practice dictates that a practitioner would not escalate therapy until confirming that the patient is receiving all prescribed medication and adherence is ensured. Of the 72 patients deemed to be on inadequate medication for their disease state, 33 (45.8\%) experienced concomitant dispensing issues. It could therefore be accepted that inadequate escalation of therapy may be directly involved in poor control in only $39(30.7 \%)$ of these cases. Nevertheless, the proportion of patients who were treated inadequately remains large, a matter that needs to be addressed. A likely explanation for inadequate escalation of therapy could be the fact that currently in the public sector, only one rheumatologist is serving the entire Free State and Northern Cape provinces. Owing to the vast number of patients attending this clinic, many patients will be seen by registrars and interns who may not escalate therapy as adequately as is required to ensure tight control.

It is therefore evident that the strongest association could be made between lack of treatment availability and poor disease control (directly or indirectly by preventing escalation of therapy), especially in the down-referral clinics. The importance of access to DMARD therapy for the control of RA is further supported by the fact that $\sim 70 \%$ of the 34 patients who were controlled reported having received all of their medication. Although determining the reasons for poor drug availability was beyond the scope of this study, the reasons are likely to be multifactorial. After interviews with pharmacists, it appears that the following may be contributing to the situation: (i) problems with budget allocation in district as well as down-referral pharmacies; (ii) staff shortages in pharmacies (most notably in district units); and (iii) issues with delivery of medication. It has also been noted that many patients often attend pharmacies outside their designated area according to the down-referral protocol. As these drugs are sent to relevant peripheral pharmacies on a 'per patient' basis, patients who are not following the correct downreferral channels will often not receive their medication. In situations where medication is not available at clinics, a patient will often be advised to return to their district hospital to receive their treatment. Two problems arise in these circumstances: firstly, these institutions often also do not have certain medications in stock, and secondly, access to transport is often problematic for many patients.

In this sample, the vast majority of patients reported good adherence to treatment. Furthermore, poor insight seemed to have played a negligible role in affecting disease control. A mere handful of patients reported problems relating to transport or administrative issues, and only $5.6 \%$ reported that adverse effects affected their treatment regimen. The reported compliance appeared to be far better than suggested by the current literature. ${ }^{[14]}$ This may have resulted from recall bias or inaccurate reporting by patients.

According to our data, $13.0 \%$ of patients had disease refractory to csDMARDs. The frequency of refractory disease may be far 


\begin{tabular}{|c|c|}
\hline & $n(\%)$ \\
\hline \multicolumn{2}{|l|}{ Gender } \\
\hline Female & $138(85.7)$ \\
\hline Male & $23(14.3)$ \\
\hline \multicolumn{2}{|l|}{ Ethnicity } \\
\hline Black African & $131(81.4)$ \\
\hline Caucasian & $21(13.0)$ \\
\hline Mixed race & $7(4.4)$ \\
\hline Indian & $2(1.2$ \\
\hline \multicolumn{2}{|l|}{ Degree of disease control } \\
\hline Remission (CDAI score $\leq 2.8$ ) & $5(3.1)$ \\
\hline Low disease activity (CDAI score $2.9-10$ ) & $29(18.0)$ \\
\hline Moderate disease activity (CDAI score $11-22$ ) & $56(34.8)$ \\
\hline High disease activity (CDAI score >22) & $71(44.1)$ \\
\hline
\end{tabular}

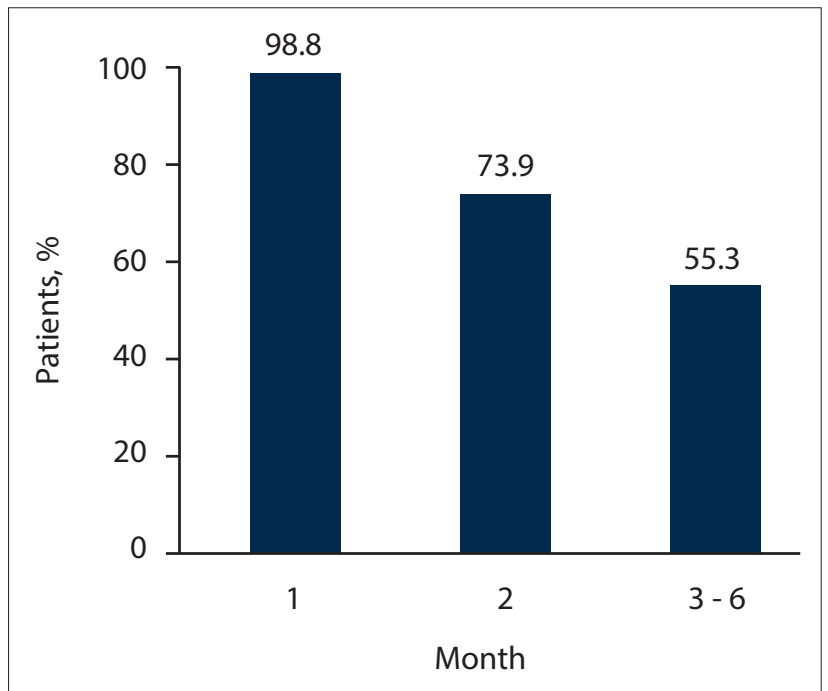

Fig. 2. Percentage of patients who reported receiving all their diseasemodifying antirheumatic drugs $(\mathrm{N}=161)$.

Table 2. Factors contributing to poor disease control among patients with rheumatoid arthritis on disease-modifying antirheumatic drugs ${ }^{*}$

\begin{tabular}{|c|c|c|c|}
\hline Reported reasons for poor disease control & $\begin{array}{l}\text { Total group } \\
(N=161), n(\%)\end{array}$ & $\begin{array}{l}\text { Uncontrolled patients } \\
(N=127), n(\%)\end{array}$ & $\begin{array}{l}\text { Controlled patients } \\
(N=34), n(\%)\end{array}$ \\
\hline Insufficient treatment for disease state & $72(44.7)$ & $72(56.7)$ & $\mathrm{n} / \mathrm{a}$ \\
\hline Dispensing issues & $71(44.1)$ & $61(48.0)$ & $10(29.4)$ \\
\hline Insufficient treatment without dispensing concerns & $39(24.2)$ & $39(30.7)$ & $\mathrm{n} / \mathrm{a}$ \\
\hline Side-effects & $9(5.6)$ & $8(6.3)$ & $1(2.9)$ \\
\hline Administrative problems & $7(4.4)$ & $7(5.5)$ & 0 \\
\hline Problems with transport/access to treatment facilities & $1(0.6)$ & $1(0.8)$ & 0 \\
\hline Lack of compliance/insight & $4(2.5)$ & $3(2.4)$ & $1(2.9)$ \\
\hline Refractory disease (no discernible cause for poor control) & $21(13.0)$ & $21(16.5)$ & $\mathrm{n} / \mathrm{a}$ \\
\hline
\end{tabular}

higher, but it is likely that a large proportion of patients who were deemed to be poorly controlled for various reasons would in fact be found to have refractory disease once these factors were attended to. However, this could only be determined after excluding other possible causes for poor control, which would not be possible to achieve in the majority of these patients. In practice, identifying these patients is important because they may require escalation of therapy to bDMARDs according to national guidelines. ${ }^{[6]}$ Biological agents are known to be highly effective in disease control, but owing to their cost, patients do not qualify until any reversible cause for poor control is addressed. A concerning finding was that of the 127 patients whose disease was not controlled, 71 (55.9\%; $44.1 \%$ of the total sample) were in the high disease activity group. These patients are at high risk of debilitating disease, and attaining tighter control on an urgent basis should be a priority.

Most of the factors contributing to poor disease control are remediable, and measures should be taken to attempt to correct these issues as far as possible. Doctors should be trained in the importance of aggressive escalation of therapy. The addition of more rheumatologists or practitioners experienced in rheumatology into the system is likely to promote appropriate management. With regard to the lack of availability of medication at peripheral healthcare facilities, doctors and patients require a better understanding of the down-referral system and staffing issues need to be addressed. After this study was conducted, the dispensing protocol has been reviewed and a central chronic medicine dispensing and distribution unit has been implemented in Bloemfontein, which is expected to improve the availability of medication to patients in this area.

\section{Study limitations and strengths}

This study had some limitations. Like all validated disease activity scores, the CDAI is subject to inter-user variability. However, considerable efforts were employed to ensure accurate scores by means of training sessions and involvement of senior doctors with the assessments. In an ideal situation, data would have been collected by one doctor, specifically a rheumatologist. Unfortunately, in this setting it was not technically possible. However, the information sheet was clear and a substantial amount of information could be obtained from source notes.

Certain data obtained were dependent on the patient's memory and therefore subject to recall bias, mostly involving the recollection of medication that the patient was not receiving from pharmacies. The majority of patients did not have their previous prescriptions available at the time of the interview. However, patients were assisted by means of visual and verbal memory aids when needed. Although a formal pill count would have been the ideal means of determining 
compliance, this was not feasible within the constraints of the study. The objective of the study in this regard was to determine to what degree poor DMARD availability was affecting disease control, and not which specific DMARDs were involved. A number of questionnaires were not clear as to which pharmacies were involved in dispensing, most notably regarding the step-down units. However, this did not affect the primary or secondary goals of the study.

Other confounders such as disease duration, baseline disease activity, erosive disease, seropositivity and acute-phase response were not taken into consideration in this study. Although these may play an important role in determining response to treatment and disease outcome, the study focused on identifying preventable factors, and the abovementioned confounders rather contribute to refractory disease. Further research is required to expand on the influence of these factors in this setting. The study also did not evaluate steroid use. Although a minority of patients end up on chronic steroid therapy, the use of these agents should be seen as 'bridging therapy' and not as a means to chronic disease control. Patients assessed in this study would have been on treatment for at least 6 months, allowing sufficient time for escalation of DMARD therapy and weaning off from steroids. ${ }^{[6]}$

To the authors' knowledge, this study is the first to evaluate the degree of disease control in RA patients in the Free State. It has contributed to our understanding of how our patients are faring on conventional DMARD therapy, and what factors need to be addressed to enable better control in this resource-limited setting where biological agents are not readily available. Once these factors have been corrected as far as possible, follow-up studies should be conducted to determine the effect of good practice on disease control.

\section{Conclusions}

Disease control in our setting is currently suboptimal when compared with other available data. Many factors contribute to poor disease control, the major concern being lack of drug availability from pharmacies (particularly from peripheral units) and insufficient escalation of therapy by practitioners. Many of these issues can be alleviated, and measures should be taken to resolve these concerns as far possible.

Declaration. This study was required for RMNS's MMed (Internal Medicine), although publication was not a requirement.
Acknowledgements. The authors thank Dr Daleen Struwig, medical writer/editor, Faculty of Health Sciences, for technical and editorial preparation of the manuscript, and Mr Mpendulo Mamba, Department of Biostatistics, for computational assistance.

Author contributions. RMNC developed the study question, wrote the protocol, assisted with data collection and wrote the manuscript. BJJvR assisted with supervising the protocol, data collection and writing of the manuscript. GJ assisted with the study design and performed statistical analysis.

Funding. None.

Conflicts of interest. None.

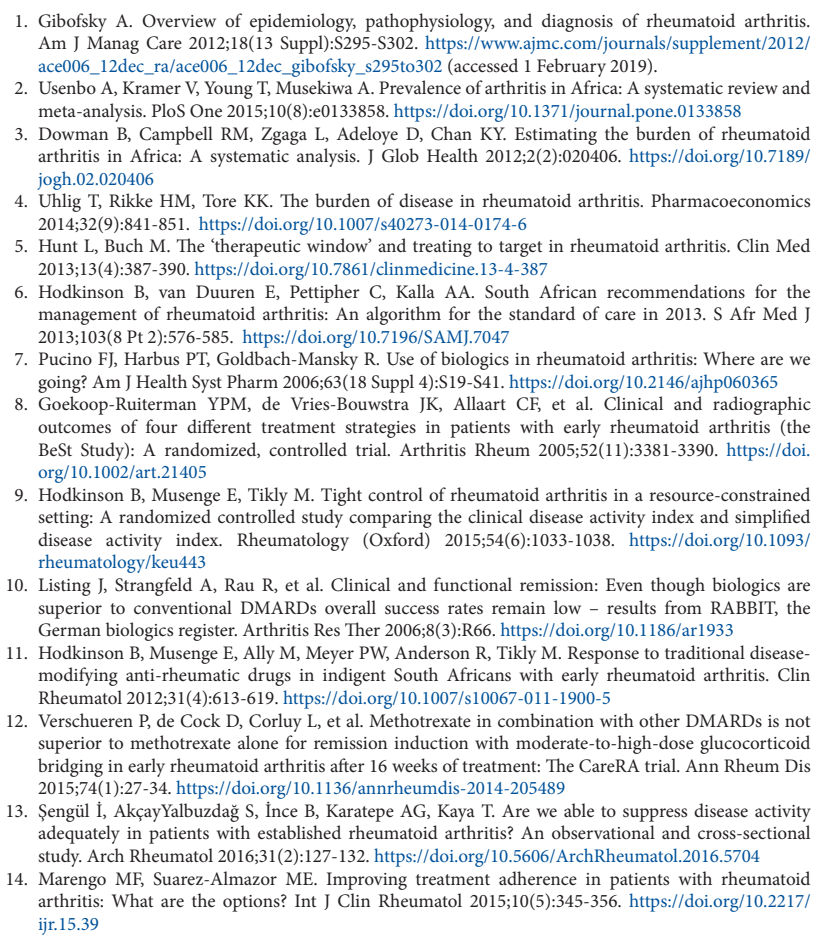

